\title{
Individual Actions as Community Informative Resources. A Collective Informative Systems Approach
}

\author{
Eliseo Luis Vilalta-Perdomo ${ }^{1} \cdot$ Rebecca Herron $^{1}$
}

Published online: 10 February 2018

(C) The Author(s) 2018. This article is an open access publication

\begin{abstract}
This paper conceives communities (in this case, partnerships) as being able to become collective informative repositories of individual and collective actions that may better-inform their members. This paper presents one approach for studying if a community has become such an informative repository. The approach used here consists of introducing a formal language (Viable Systems Modelling, VSM) into one of the community nodes (a participant) and tracing if its use is seen in another node (another participant) - indicating the presence of a process of diffusion. This research design has been tested in a crimereduction partnership in the UK. One of its members was asked to engage in the design and testing of this approach as a co-researcher. As a result, a questionnaire to map communication and control devices inside an organization was jointly developed. In keeping with VSM principles, the questionnaire encouraged participants to reflect on attenuation and amplification processes within their communications channels. To test the quality of the outcomes of this approach, members from another crime-reduction partnership were also invited to answer the survey; this was to confirm that VSM notions were not evident for those outside the development and testing of the questionnaire. The questionnaire indicated also its capability to make visible communication and organizational processes within collectives and its potential to stimulate self-organization, for those individuals who became familiar with VSM. Furthermore, this approach provided the authors with the capability to study information flows inside the two collectives, and contributed to an understanding of these flows as a model for building and maintaining a Community Informative System.
\end{abstract}

Keywords Collective informative systems · Individual action · Collective improvement · Viable system model

Eliseo Luis Vilalta-Perdomo

evilaltaperdomo@lincoln.ac.uk

Rebecca Herron

rherron@lincoln.ac.uk

1 Lincoln International Business School, University of Lincoln, Brayford Pool, Lincoln LN6 7TS, UK 


\section{Introduction}

This paper reports on an experiment conducted to test the functionality of a research approach designed to study if a community (in this case, a partnership) could be supported to act as a collective informative repository of individual and collectives' actions, that may better-inform its members. To study, through scientific lenses, how to inform individuals on acting collectively in an effective and efficient way, seems to provide certain advantages when striving for long-term operations. The trigger for such research is the observation that for much of human history communities did not have at their disposal many formal ways for theorizing on how to organize collective actions to increase individual viability. Pyramids were of course built, and empires ruled, but little formal analyses were developed to identify which collective actions were better or worse from the perspective of the individuals involved. Divinely-inspired behaviors or cultural uses and taboos were local approaches used to guide choices of action, but the results from these procedures could not easily be generalized. Even today, the validity of this kind of advice seems deceptively problematic.

It is certainly not new to claim that science applied to management may maximize prosperity and makes it permanent (Taylor 1911), or that it can provide relevant knowledge to support problem-solving or decision-making in ongoing enterprises and institutions (Kast and Rosenzweig 1985). This view suggests the ability of individuals to define criteria to recognize which collective actions are better for themselves, even under cognitive limitations and time constraints (Simon 1957). However, this ability to recognize and agree criteria does not always seem to be the case. Top-down collective actions are not always beneficial to everybody; for instance, governmental authorities may remove houses in exchange for expected public interests, such as building roads. In these cases, trade-offs need to be negotiated and made explicit. As explained by Arrow (1950), individual preferences are then removed and a collective one, decided by the few for the rest, takes precedence. The most immediate consequence of this is an impoverishment of potential avenues for action, and of latent resources.

Other theoretical approaches suggest that the internal organization of individual interactions may result in an effective management of collective resources. For instance, Ostrom (2009) and Skyrms (2007) provide alternative evidences to characterize the kinds of rules that may support self-organization; even though they do not explain how such rules can be developed. In this paper, we continue building on this particular track, by recognizing collective research activities as resources that trigger individuals' actions in a collaborative environment; this to rise the variety and amount of resources available and to improve participants' own and others' performances. The aim is then to increase an individuals' propensity to become a resource for other individuals and vice versa. We posit that this approach increases individuals' commitment to collective activities and therefore strengthens communities' viability.

It is important to notice that in this paper we will be using indistinctively the terms collective and community, and we will understand both as: "a number of persons having certain beliefs in common and moved by widely shared habits of feeling and judgement" (Dewey 1948:199). We will also understand improvement in a general sense; as a way to increase the viability of organizations (Beer 1972).

The research reported here started approximately a decade ago when a new legal requirement was introduced in the UK that all the activities and decisions of local bodies (such as Local Government Authorities and Police Authorities) needed to consider the impact they 
would have on the reduction of crime and disorder. For conducting this collective research, we selected two newly-formed crime-reduction partnerships located in two industrial English towns within nearby regions. As well as studying these in a traditional sense, we posited that by looking at these collectives as an informative source for their members, we might additionally be of help moving them from a largely passive observation of their behaviors to more active engagement intended to foster increased individual self-awareness and collective self-organization. As we will explore more in detail below, in one of the cases we used a formal systems language, the Viable System Model, VSM (Beer 1972), to introduce a new vocabulary within their partnership, and then create a research tool that enabled members of this partnership to use central concepts from this analytical tool. In the other case we just observed their activities without any deliberate intervention in them. The reason for choosing to use VSM for this relates to its very strangeness in every-day life. VSM concepts, such as 'attenuation' and 'amplification' are relatively alien to many people, and have even been seen as 'cumbersome' by some authors (Hoverstadt and Bowling 2002). As such, finding their use in subsequent business conversations within a research project would therefore suggest that their users had been exposed to the others' influences through the course of their conversations.

Furthermore, and as importantly, as by-product of the use of VSM, it was expected that it may help people influenced by this language in reflecting on their own activities and the way they communicate and coordinate such actions to others, and vice versa. For example, in the other partnership, where VSM remained inaccessible, we observed a more passive, traditional manner of developing strategies; it therefore played a role as a kind of 'control experiment' for us to compare and to discuss the outcomes from both partnerships.

In summary, the theoretical contribution of this paper is linked to the formalization of a particular challenge: To identify if collective research activities may support the creation and maintenance of communities (collectives), that behave as collective information repositories, able to better-inform individual actions, in such a way that both, the (new) members and the (newly-constituted) collective, may improve their performances.

\section{On Studying Coordinated (Informing) Action}

Doing something to trigger the actions of others seems to be a simple exercise. There are many examples where, after triggering an individual action, we can observe collective behaviors. But the questions often remain - are these highly-coordinated behaviors and do they translate into an improvement of the participants' performance over time? For example, if we throw a ball to a group of children, we can reasonably expect them to start playing some sort of game, but would not necessarily expect it to be a fully-regulated football match. Would their play be viable and continue over time? Knowing how to actively create a coordinated outcome (for example how to create a winning football team over time) requires something related to the quality of that which triggers individuals to excel in their actions in a coordinated way; in other words, it requires us to somehow communicate which individual actions carry collective information available and useful for everyone. This appears to be one of the fundamental distinctions between getting advice and acquiring knowledge (Fischhoff 1992). In this research, we are asking what supports not only ephemeral individual and collective actions (such as fans singing in football matches) but what may be likely to happen beyond chance 
events alone. One possibility is to work at developing collectives constituted by individuals informed on how they contribute at their best; collectives that carry information and, therefore, that behave as if they were a Collective Informative System (Coll-IS).

The rationale behind the use of 'systems ideas' over other possible ways of describing this research, such as an ethnographic approach, is the assumption that systemic elements (i.e. the partnership boundary, its members and their interactions) can be engineered, to enable participants in following their particular interests and preferences in a cooperative environment. This is labelled by Churchman (1971) as a 'Singerian Inquiring System' (SIS). SIS is characterized by not having a terminating point and, as it is the case of 'continuous improvement' processes, it develops around tensions emerging from discussions on what a system 'is' vs. what it 'ought to be' (Ulrich 2005). This dialectic process may provide their users, in our instance the partnership members, of enabling information to achieve their aims. In this context, we understand Coll-IS as conversationally-built action-oriented repositories that help individuals to engage in long-term coordinated actions, which may support improvements to individual and collective performances.

There is, however, a dynamic challenge emerging in this kind of exercise. Collectives are not static entities, new members may join while others will defect, which brings new challenges. Constraints can be used to control interactions to generate and restrict the variety needed to construct collective actions independently of any particular individual (in much the same way the Football Association now regulates the game of football; for most part independently of any given individual). For the collective of actors to behave in a democratic and ethical way, we would consider these actions to be desirable if they maintain the collective open for other actors to join in. Subsequent contributions increase collective's capability to deal with external sources of variety, therefore supporting and possibly improving the collective action. Von Foerster's (1999) criterion will therefore be of value to promote these interactions: what is desirable is what does not restrict the variety of actors who are not supported (yet).

To achieve a desirable coordinated performance from individual actions, demands analyzing the presence of Coll-IS at two levels. On the one hand from the individual view, one may look at someone informing her own actions for her own personal benefit. On the other hand, the collective approach considers members from a community who can agree on their preferred behaviors, and use these to orient their individual actions. The former perspective is the field for traditional operational research (i.e. hard OR), where expected outcomes are identified through analyzing data to inform decision-makers or solving problems. The latter procedure has been studied in detail through System Thinking approaches like SSM (Checkland and Holwell 1997). Mixed approaches have also been examined (Espejo et al. 1996). Interestingly enough, there is still space for further reflection on cases when there may be explicit negotiation between members of a particular collective, but no overall aim is required, nor consensus agreed; where individuals keep their links with other members in a community due to their personal commitment and interest (Sen 2005).

According to the knowledge management literature this has been tangentially discussed in terms of 'Communities of Practice' (CoP). Lave and Wenger (1991) recognize the existence of distinctive knowledge interwoven to particular social practices. To become members in a specific community, individuals should learn the practices of such community, as it is the case inside institutions. This implies the recognition of distinctive activities that have been developed through years of practice, like those that constitute professions. However, our task is one 
of a more general nature. Our aim is to study how to increase individuals' propensity to create and maintain such communities. We posit that by doing so it would be possible to begin to unravel the inherent complexities of self-organized systems.

\section{Describing the Research Problem}

The main difficulty we found in doing this research is part of a very old problem, how to map interactions between individual and collective performances; which individual actions contribute to the collective, and which forms of collective action inform individuals on how to behave. The design of research interventions assumes that it is possible to link one to the other and vice versa. For instance, a certain class of individuals, such as leaders, may be seen as agents of collective change. Furthermore, communities such as religious orders change the way individuals behave. Accordingly, one option to study these relationships is to map them in stable communities. However, as our original problem looked at the creation of new collectives, or at least the development of newly-formed collectives, studying leadership or institutional life would not be sufficient. The reason for this lies in the fact that inside stable collectives it seems difficult to draw distinctions between both levels of interaction, the individual and the collective, at the same time (Spencer-Brown 1972). In general terms, it is expected that the collective would inform the individual, but not so much the other way around. Communities of practice literature assumes that it is the membership who must learn how to behave, if they want to stay inside the collective, and new ways of interacting are not necessarily expected or promoted. This is an example where interactions are institutionalized, and the collective becomes an institution (Searle 2005).

One alternative way to study community-building and development is to wait until several individuals decide to do things together and to observe their progress. Even though this would be an appropriate way to conduct this research, we could not follow this road, as time was a pressing resource. Another possibility, the one preferred here, was to observe and participate in the development of two relatively new groups that were not at that stage particularly stable, but aimed to be, and to map how individuals' conversations constituted an emergent collective identity and vice versa. By following this procedure, we could conduct an ethical study that aimed to support the success for the constitution of a collective, whilst respecting individuals' own perspectives. No explicit external impositions about values, expectations or norms were present; except for a general expectation that individuals were striving for the creation of a collective capable of supporting its members.

\section{Research Strategy - Two Examples from UK Crime Reduction Partnerships}

The possibility to explore and test these ideas became a reality when the Authors were invited to investigate forms of collaboration and information sharing between different stakeholders involved in a program focused on reducing crime and disorder inside neighborhoods. The program, initiated by the UK's Home Office, was based on the implementation of the Crime and Disorder Act (1998). From its origin, the aim of this initiative was to reduce crime and disorder and to help create 'safer communities' (Home Office 1998). 
The program focused on prevention, with 'swiftness of response' (to reduce crime) being its key performance criteria. It was also seen as important to focus collective participation (i.e. that of the local government/community partnerships) on reducing crime. And finally, to raise local awareness, it required the inclusion of information about crime and disorder in all local government decision-making processes. The implementation of this program in two English towns was the occasion to observe and test our initial research interest, how to study what informs individuals' actions from a collective perspective, and how these actions could become a collective resource that contributes to their viability. Our interest was also to explore which kind of informative device could support peoples' interactions and, at the same time, how the latter could create the former. This suggests a degree of circularity in the process.

The government program required the development of a three-year strategic plan based on the three premises previously stated: prevention, participation and better-informed decisionmaking at both the individual and the collective levels. This implied two tasks; firstly, to inform individuals from different governmental and community organizations, and secondly, to observe how they determined and implemented collective actions that further informed members inside their community.

The innovative part of this research is that the authors used the research process itself as a trigger for the participants to become active knowledge producers and repositories of it, both in the research and in the development of their collective. This involved them behaving as 'research actors' rather than 'actors in the research play'. The researching strategy was then designed to encourage participants to make their own observations and develop their own language and coordination systems - á la Ostrom (2009). To observe these informed changes in the collective, we introduced linguistic markers that could trace changes in their interactions (with the participants' consents). Several communicative structures could be used for this task; for instance, we could have used the natural language used by participants already: English in this case. However, the idea was to trace changes, hence the design involved the presence of 'alien' words in interactions inside the collective; words that would not come from a coincidental exposure to other random interactions from the environment: such as the TV, books or other individuals from outside the partnership. Hence, the rationale behind the selection of such alien language involved two main characteristics: 'strangeness' and the inherent capability for self-reflection on the quality of communicative devices inside the community. Systems Thinking literature proposes several languages of this kind, but as our aim was to increase individual and collective viabilities we selected the Viable System Model (Beer 1972). This language seems to fit the purpose well as it suggests the production of autonomous systems inside autonomous systems (Espejo 2000). In principle, the notion of an autonomous system (individual) within an autonomous system (collective) seemed to be sufficient and sufficiently constraining to observe actions at different levels. An additional advantage in the context of this research is that the language of VSM has been described as complex and difficult to implement, even sometimes characterized as 'cumbersome' (Hoverstadt and Bowling 2002); for instance, particular words from the VSM language, such as 'attenuation' or 'amplification' used to characterized communication channels, are not common inside the business parlance. Interestingly, for this piece of research, the use of VSM suggests its convenience to act as a linguistic tracer inside the partnership's conversations, and would also provide space to identify the alignment of individual purposes to constitute a collective one, if that was found to be present. 


\section{Research Design \& Implementation}

In designing this research study, two crime reduction partnerships (Partnerships A and B) were chosen to act as comparative cases. Both crime reduction partnerships (A and B) were responding to the new legislation and developed ways of sharing information about crime reduction across local authority partners in their own very distinct ways. These cases gave us the opportunity to use collective research as the trigger to support community development in Partnership A, and to compare these outcomes with activity in Partnership B.

In Partnership A, the research design was based on studying a new collective that was striving to become a stable community through what we posited was a self-organizing approach. This case concerned a Community Safety Partnership established in 1998, constituted by representatives from the County Council, Police, social services and several other organizations, such as: Association of Neighborhood Watch Groups and Pub \& Club Watch; churches from different denominations; A gay and lesbian community group, and The National Farmers Union, among many others. To face and tackle crime the partnership proposed (a) a systematic program of proactive and reactive security improvements of domestic dwellings (known as target hardening), and (b) the intensive supervision and monitoring of the most prolific and persistent offenders.

Partnership A was selected due to their use of a human-based approach to enabling information to be shared and discussed among interested and relevant parties. One example of such an approach concerns a program established in 2003 related to community activities against crime (Program OCAC). This program focused on crime and drug use, and consisted of a set of representatives (of the different organizations listed above) looking together at changing trends, sharing information and ideas, and commissioning drug and alcohol treatment services as measures to reduce crime. It also involved them working together with residents and other voluntary groups on specific neighborhood problems, to improve how existing services were delivered and to encourage young people to take up useful leisure activities. The partnership met face-to-face on a regular basis to discuss and develop activities. It was during these meetings that we began to map their communicative structures and investigate if there were any possibilities for knowledge transfer among the partnership members.

Partnership B concerned a Community Safety Partnership, also constituted in 1998 by representatives from the District Community Safety Partnerships representing the seven District Councils inside an English county. Each District Council was the representative for the different organizations and initiatives that where taking place inside their areas of influence, and in turn they reported such activities to the Partnership. Instructions emanating from the Partnership were communicated to the different organizations and initiatives through the District Councils. This role as representative suggests that this Partnership was built through a top-down approach. The rationale behind selecting this Partnership was to use it as a group control to see the differences in the evolution of their communicative patterns, if any. In Partnership B, the object of study was a project (called "Project JinL" from now on) concerning the development of computer-based tools to analyze crime and disorder data for better-informed actions against crime. As a way to ensure effective exchange of crime and disorder information, Project JinL was established to gather, interpret and disseminate information. Accordingly, Partnership B consisted of the development of a formal Computer-Based Information System (CBIS). This CBIS took the form of a Geographical Information System 
(GIS) as a source for additional authority, able to produce and make available maps about crime and disorder as fundamental information devices for developing anti-crime strategies. Observations collected about this partnership were focused, firstly, on recognizing how people involved in the project engaged in producing the required relationships with other partnership members; secondly, on identifying strategies to guarantee that the resulting collective would be effective given time. In a nutshell, Partnership B members engaged in technical discussions on how the different actors involved could be linked through information sharing.

For Partnership A, we wanted to map how different members of the partnership communicated between themselves, and to recognize if there were any knowledge transfers. To do this, three different activities took place. First, three partnership meetings were observed and notes taken at each. Second, three interviews were conducted with members of the Partnership: The Community Safety Partnership Coordinator; a Senior Probation Councilor, and a Senior Probation Officer ('Mr. SPO' from now on, who played a fundamental role in this research). Finally, with the aim of tracing potential knowledge transfers, the research design included a collective activity, where a single member of the Partnership (Mr. SPO) was introduced to the VSM language. The intention was to jointly develop (between Mr. SPO and us) a questionnaire concerning the ways the Partnership established their strategic plans. The rest of the participants (from both cases A and B) would answer this questionnaire, and by doing so we (the authors) would check if VSM concepts would still be alien to members in Partnership A or if they had been successfully incorporated as part of the language used in their interactions. If members of Partnership A did not need any assistance to answer the questionnaire, it would suggest a successful knowledge transfer between the questionnaire developer (Mr SPO) and the others. Questionnaires were also filled by members from Partnership B, to confirm that they were unable to answer them without the assistance of one of the authors of this paper; as this was expected due to the complexity involving the use of the VSM language.

In a nutshell, the design approach for this research considers using research to do research, and involves the use of a circular process where a collective shows its capability to observe and consider its own activities in a more abstract and formalized manner; abstract due to the use of concepts pertaining to VSM and non-familiar in their natural language (English in this case), and formalized as their communication could be map in terms of such concepts. The presence of both capabilities, developed internally, suggest a research design able to support selforganization.

Participants and their roles from both Partnerships, A and B, are introduced in Table 1

Table 1 Participants and their roles from both Partnerships

\begin{tabular}{ll}
\hline $\begin{array}{l}\text { Partnership A. Example of 'collective informative } \\
\text { system' (CIS). }\end{array}$ & $\begin{array}{l}\text { Partnership B. Example of 'formal } \\
\text { computer-based information system' (CBIS). }\end{array}$ \\
\hline $\begin{array}{l}\text { Questionnaire developer } \\
\text { - Probation Service, Senior Probation officer }\end{array}$ & $\begin{array}{c}\text { Questionnaire developer } \\
\text { - None } \\
\text { Questionnaire respondents }\end{array}$ \\
$\begin{array}{l}\text { - Community safety Partnership, Co-ordinator. } \\
\text { This position was occupied by two different people } \\
\text { during the duration of this research } \\
\text { - Probation Service, Senior Counsellor }\end{array}$ & $\begin{array}{l}\text { - Project JinL, Principal Research Officer } \\
\text { - Police, Data Officer }\end{array}$ \\
\hline
\end{tabular}

Note: All the participants were interviewed at least once during the duration of this project 
Table 2 Research design and implementation

Stage 1: To identify how to use linguistic traces to identify interactions that create collective knowledge

Task 1.a - to identify who constitutes the collective; to draw its boundaries

Task 1.b - to find the different communicative protocols (existing or required)

Task 1.c - to select different potential joint actions

Task 1.d - to explain the Viable System Model and jointly develop the questionnaire

Stage 2: to develop an observational tool (questionnaire)

Stage 3: to test the stability of the questionnaire by an application to a sample

Stage 4: to apply the tool to some additional members

Three participants from Partnership A were involved in different parts of the research design and implementation, which consisted of four stages (see Table 2).

Stage 1 - To identify how to use linguistic traces to identify interactions that create collective informative resources (collective knowledge).

This stage involved a set of tasks. The first task consisted of a series of conversations to collect observations about the creation and maintenance of the collective: what and who constituted the collective, and where to establish its boundaries. In Partnership A, this step consisted in behaving as a passive observer in three meetings that involved defining the Program OCAC. These meetings included representatives from the agencies participating in the program that consider OCAC pertinent to their activities. Observations on the interactions between members suggested an extended agreement in terms of their organizational boundary. According to their descriptions, people interested on participating just had to appear in their partnership meetings to be considered part of them, with the freedom to engage through different levels of commitment. In contrast, for Partnership B interviews were conducted with the main two actors of Project JinL (Research Coordinator and Principal Research Officer). They made explicit that the participation in the project was on the basis of a more formal topdown internal selection process.

The second task involved observing the efforts members made to maintain their collectives. The aim was to identify the different protocols Partnership A and B developed and used for this purpose. This allowed us to recognize different instances of coordinated actions. As commented above, in Partnership A, interviews were conducted with three members of the partnership actively involved in OCAC (The Community Safety Partnership Coordinator, a Senior Probation Councilor and Mr. SPO; see Table 1, Partnership A). Interviewees made explicit that no formal ways to interrelate were in place. Partnership members expected that new practices and protocols would result in activities that could facilitate future stability for collective action. It was thus of great interest to see if we could observe any evidence of this. Their main concern was not about 'being able to act together', but being able to act in ways that could be considered effective and efficient by those involved, and ultimately by others observing the partnership from outside; an example of this was the program OCAC. In this context, we hoped to provide some additional support, through the research process, which would help members of the partnership to transform themselves from being the observed to become informing observers, people able to recognize why certain decisions were preferable to others, and to help other members in doing so.

We planned to engage members in Partnership A through reflective action (Schön 1991); an activity which would help them to recognize themselves as a Collective Informative System (Coll-IS). The main assumption was that people's actions may be mapped by following their 
conversations and information exchanges (Beer 1985). Accordingly, the aim of this exercise became the introduction of a common (systemic) vocabulary which would help those involved to become a collective capable of producing new distinctions that helped to maintain themselves together doing something. It was expected that through this action, knowledge might be created, maintained and transferred.

The preferred research action with Partnership A was to develop an observational tool jointly with a member of the partnership; one able to look specifically at information flows within the partnership. This provided possibilities to map simultaneously individual and collective action. The Senior Probation Officer (called "Mr. SPO") was identified as the best candidate to participate in the development of this observational tool.

The procedure supported the introduction of certain notions into group conversations and consisted of several steps. First, with the support of Mr. SPO, we explored together how autonomy can be developed inside a self-organizing collective. Discussion followed VSM notions and where around the idea of how to stimulate the ability to create, produce and regulate their own meanings within a collective (Espejo 2000). In the language of Viable Systems Modelling, linguistic protocols can be analyzed in terms of devices for variety engineering: amplifiers and attenuators (Beer 1985). Therefore, to observe any evidences of self-organization, we (Mr. SPO and one of the authors) established a general procedure to consider the collective's processes: (a) how they decide their objectives; (b) how they produce these expected objectives; (c) how they evaluate the current results, and (d) how they evaluate other future or unexpected outcomes. Each of these elements were explored to articulate protocols that amplify or reduce the collectives' communications. The summary of these different formal protocols relating to self-organizing collectives are presented in Table 3. This table is an illustration of the framework used to design the questionnaire itself. Each of the above theoretical elements was expanded into a sentence with an accompanying list of 'protocols' that highlighted the different forms the information flow could take.

Table 3 Protocols to highlight the different forms the information flow could take (adapted from Beer 1985)

In the process of deciding the objectives of the collective:

-Protocols that amplify the communicative impact, in that they are generated to ensure all members of the collective are properly informed about expectations

-Protocols that attenuate differences within the collective, in that they are designed to achieve a shared vision among their members

In the process of producing the expected objectives of the collective:

-Protocols that amplify the communicative impact, in that they are designed to improve ways for interchanging information among members inside the collective

-Protocols that attenuate differences in the collective, in that they are designed to filter unexpected events during their performance

-Protocols that coordinate the activities in the collective, in that they are designed to maintain active links amongst their members

In the process of evaluating results of the collective:

-Protocols that amplify the communicative impact, in that they are designed to promote individual and collective reflection among the members of the collective

-Protocols that attenuate differences within the collective, in that they are designed to recognise inadequate performances at individual and collective levels

In the process of evaluating other outcomes of the collective:

-Protocols that amplify the communicative impact inside the collective, in that they are designed to recognise future opportunities and sources of risks

-Protocols that attenuate differences within the collective, in that they are designed to recognise unexpected outcomes at individual and collective levels 
Once the set of protocols were linked to the VSM model, it was important to find meaningful ways to use this within the group. We were much aided in this by other two partnership members who offered to participate in extended interviews and data collection with us.

\section{Stage 2 - $\quad$ To develop an observational tool (questionnaire)}

As expected from any application from the stables of 'scientific method', we wanted to look for the repetitive or repeatable (Ashby 1956/1999). Work was oriented at ordering and classifying individual observations that might be shared and used by others; therefore, becoming high-quality observations (de Zeeuw 2001a). However, this is not a simple issue when the research intention is also to empower the (people) observed to become informing observers. In this event, the main challenge was to organize efforts in such a way that these efforts would also contribute to the process of the research itself. In Partnership A, protocols were identified but no specific interfaces were developed, yet. Meetings were scheduled, (and coffee and cookies served), but what to discuss in them and which were the expected outcomes were not defined a priori.

As outlined above an initial questionnaire associated to VSM was developed before the meeting with Mr. SPO (see Table 4). The questionnaire involved mapping some VSM elements (systems 1 and 2 in Table 4).

Through face-to-face conversations with Mr. SPO certain notions were introduced; notions aimed to support individual reflection. As we said earlier, our selection involved

Table 4 Questionnaire elements mapped from VSM (systems 1 and 2)

1. Understanding about System One (as many as activities)

1.1. Transformation

1.2. Management (Identify autonomy at their level and below)

1.2.1. Creation

1.2.2. Production

1.2.3. Regulation

1.3. Communicative Devices

1.3.1. Amplifiers

1.3.2. Attenuators

1.4. Relations among different activities (squiggly lines)

2. Understanding about System Two (Coordination)

2.1. Anti-oscillatory mechanisms

3. Understanding about System Three (Control)

3.1. Intervention (Rules)

3.2. Resource Bargain

3.3. Accountability

4. Understanding about System Three* (Audit, Monitoring and Control)

4.1. Measurement and indicators (Identify the process of definition also)

4.2. Procedures for auditing

5. Understanding about System Four (Intelligence)

5.1. Environmental scanning process (Actual and unknown)

5.2. Planning process

5.3. Production of the model of the Collective

6. Understanding about System Five (Policy-Making)

6.1. Issuing of policies

6.2. Monitor relations between Decision-Making and Intelligence

6.3. Algedonic signals 
the use of Beer's Viable System Model as it can characterize organizations in terms of (a) primary activities, (b) environment, (c) variety and (d) management (Beer 1985). We expected to see traces of these conversations later when talking and applying the questionnaire to others.

To enable this, with Mr. SPO's help, we constructed a linguistic framework (machine) able to generate questions. The intention of developing this machine was that if used directly with participants, it would help to distinguish between observations, and order these following protocols previously proposed in Table 3 . This machine would generate relevant questions able to convert elements of Tables 3 and 4 into aspects participants could reflect and thus answer to, albeit with the need for the interviewer to explain the meaning of some of the terms. Initial ideas for building this machine were taken from 'facet theory' (Guttman 1959; Shye 1978), mainly the notion of 'mapping sentences', a tool for generating observational strategies. The introduction of the machine allowed the different elements to be systematically explored in different combinations, and the result was a tool able to generate a range of questions, but at the same time engage the respondents inside the process of researching and reflection on their own activities.

Table 5 presents a schematic of this machine. It shows the constituent parts that could be selected to generate a relevant question concerning the theoretical constructs. To generate a question each box was selected, and choices made from the box where multiple options were possible. This provided a sentence, a statement about the collective, to which the respondent was asked to indicate how often they felt this was the case. This involved using a Likert scale (i.e. 'always', 'usually', 'sometimes', 'rarely' or 'never').

Table 5 Machine for generating relevant questions with researcher-participants

An example of the potential combinations available and questions produced is as follows:

- The combination: 'creation / others' meanings / externally defined / amplifiers / intervening / results'.

- Becomes the following question: The expected results of the collective and their operationalization are defined by superior authorities - e.g. Home Office. These expectations are informed to their members through specific governmental procedures and regulations. These are focused on amplifying their impact by means of new technologies -e.g. internet, e-mail.

- With an answer selected from: 'always', 'usually', 'sometimes', 'rarely' or 'never'.

Table 5 Machine for generating relevant questions

\begin{tabular}{lll}
\hline $\begin{array}{l}\text { Members of the } \\
\text { collective participate } \\
\text { in the }\end{array}$ & $\begin{array}{l}\text { Creation production } \\
\text { of regulation }\end{array}$ & $\begin{array}{l}\text { Others' meanings, } \\
\text { their own meanings, }\end{array}$ \\
as they co-ordinate among themselves through & $\begin{array}{c}\text { externally defined internally } \\
\text { negotiated }\end{array}$ & mechanisms, \\
$\begin{array}{l}\text { they make use of a(n) } \\
\text { and audit their performance in terms of their } \\
\text { always. } \\
\text { usually. } \\
\text { sometimes. } \\
\text { rarely. } \\
\text { never. }\end{array}$ & $\begin{array}{l}\text { decision-making process, } \\
\text { results understandings }\end{array}$ \\
\end{tabular}


Stage 3 - $\quad$ To test the stability of the questionnaire by an application to a sample

In the third stage, another participant from Partnership A (the Community Safety Partnership coordinator) was interviewed in two more occasions using this questionnaire to facilitate discussion and generate data with a cross-section of participants. These interviews indicated no presence of VSM language yet, but where useful to allow individual reflection on the way members inside the Partnership acted and communicated collectively.

Therefore, to achieve the production of a fully-usable research tool required several attempts, and Mr. SPO helped us to rephrase the questions and reorganize the questionnaire following VSM concepts. Elements of effectiveness and efficiency were used as operational criteria for developing the tool; effectiveness in the sense of providing the interviewees with enough distinctions to satisfy their requirements to develop and describe the collective; efficiency in the sense of reducing as much as possible the resources required for achieving the task (e.g. time from interviewees, and rounds of interview). After three lengthy iterations, a final tool was developed. Examples of different questions can be seen in Appendix 1.

\section{Stage 4 - $\quad$ To apply the tool to other members in the community}

Once this process was refined and a shorter final tool developed, the questionnaire was applied to other members of the Partnerships A and B through one-to-one interviews. The aim was to collect data on the functioning of the collective, to recognize also the potential presence of a process of diffusion, and if so, to recognize if such diffusion took place only in Partnership A or was something beyond this exercise. The presence of diffusion in Partnership A was positive, it would suggest that the collective itself was behaving as a Collective Informative System; a repository of knowledge, transferable throughout the community. In order to trace such process of diffusion, keywords were used; these came from the VSM language. The preference for the VSM language rather than a natural language, such as English, was because of its peculiarity. The strangeness of VSM had the benefit of being easily recognizable in many different contexts. In Partnership A, it was effortless to notice that participants were learning unfamiliar words and their associated concepts. It is interesting to notice that the presence of this diffusion process could also be used to evaluate the level of informative health inside the collective; the quality of a network in terms of accuracy communicative closure and speed. However, this was out of the scope of this research.

The questionnaire was applied to two members of Partnership A that had not seen it previously (a Senior Counsellor at the Probation Service and a Data Officer from the Police), and two from Partnership B. This activity was useful: (a) to test if the questionnaire would help them to generate observational data about their own situation, and (b) to encourage them to reflect on and respond to some of these issues. Members from Partnership A showed understanding on concepts related to 'amplification', 'attenuation' and 'coordination'. This suggests the collective was behaving as a Collective Informative System (Coll-IS) through (a) the collection of evidences of self-organizing protocols described through the question generator and (b) the observation of the diffusion of ideas through the collective, evidenced by the use made of the language of Viable Systems Model in the questionnaire. Conversely, no understanding was present in the control group (Partnership B), and filling the questionnaire was a difficult exercise to accomplish, as continuous explanations were required. Furthermore, 
this exercise showed that the machine was a useful tool for generating systematic and thorough questioning and to provoke self-reflection.

It is important to notice in this research that observations of Partnership A also include previous parallel evaluations the University team was undertaking. Other research activities concerning Partnership A focused on investigating their forms of collaboration and information sharing between different stakeholders. These activities indicated also that their members were repeatedly acting in a self-organizing manner. They exhibited behaviors and initiated activities that were not simply showing compliance with the new legal requirements but were producing new activities the collective itself found valuable. For instance, the Police, the Probation Service and the Local Authority were implementing projects to deal more effectively with prolific-offenders and to improve the security of the homes of robbery victims. Individuals from different organizations voluntarily entered and contributed to these activities, as part of their own interests in understanding and presumably improving their own actions. As such, we are also mindful of the interest shown by participants from Partnership A in becoming 'coresearchers' and the value of this to this kind of study.

Now, more than ten years later, both Community Safety Partnerships continue operating. It can be argued that the main reason is they are legal bodies responsible for bringing together organizations concerned with reducing crime, disorder and anti-social behavior in their council areas. However, there are noticeable differences between the different activities each Partnership currently runs. On one hand, Partnership A shows in their website concerns on partnership and community development. This is indicated by different programs developed between the partners. These are: Neighborhood Watch Group Network, a community of over 150 Neighborhood Watch Groups; Neighborhood Action Teams, to discuss community issues and seeks to resolve problems; Safer Neighborhoods Operations Group, to deal with the overall delivery of the Community Safety Plan; CountryWatch, to work closely with rural communities to tackle farm thefts, poaching, badger baiting, etc.; Horsewatch, to support horse owners across the local region aiming to provide advice and information to assist in the prevention of crime, and Community SpeedWatch to provide speed signs for local Parish and Town Councils to hire in an effort to tackle this problem. On the other hand, Partnership B continuous showing in their website a top-down approach as they describe themselves as a 'statutory body'. When looking at their website no collective activity is presented; what it is available is a Community Safety Agreement for 2015-2018 focused on explaining the Partnership structure. However, a particularly notable outcome from the project JinL in Partnership B is the development of a 'Research Observatory' that provides data about the County to people interested in any of the following themes: access to services; crime and community safety; deprivation and poverty; economy; education and skills; environment; health; housing, and population. This is in itself of considerable value.

\section{Reflecting on the Research Strategy}

In this research, we suggest the possibility to identify protocols inside collectives that inform individual activities from a collective's viewpoint and, at the same time, build the latter from the former. We claim that these protocols may also be developed and implemented by involving the participants as active researchers. Accordingly, rather than seeking to develop general descriptions, the research focuses on proposing and testing four instructions, stages 1 to 4 , useful to enrich the information available in the interactions between members of the 
collective. These guidelines suggest ways for improving both individual and collective performances. In this context, we extended the traditional understanding of knowledge systems, to include "a group of people maintaining and developing a collective that behaves like an [informed] actor" (de Zeeuw 2010).

Fortunately, there is relevant literature developed to support these kinds of protocols in terms of attenuators and amplifiers that might control and monitor interactions (Beer 1985). We also look for inspiration at other notions such as (a) evolving cooperation (Axelrod 1990), (b) propensity exercises (Rapoport 1988), and (c) the development of strong actors (de Zeeuw 2001b). However, testing these protocols is not an easy task because it demands individuals acting inside existing communities where collective actions can be observed and high-quality descriptions be built. The challenge seems to be then how to identify communities, (or build them if there are none available), where individuals engage in collective actions, useful as resources for any individual rather than for one in particular. Following the ideas of Luhmann (1984) and Searle (1996), we identified the opportunity to reinforce the development of a social system by introducing a structuring task and a supportive language into their conversations. The task had the intention to involve members of the partnership in a joint activity that would make them to interact in new ways. The language also had the aim to identify if there was any propagation of ideas through their collective task; to do so the language was introduced to one of them, and was expected to be identified by others who were initially unfamiliar to it.

In the previous context, the quality of the knowledge acquired was related to the impact on the collective's language capability. This approach elaborates beyond traditional approaches for encouraging or inducing individuals to act more effectively and efficiently. Our research design works around the presence of linguistic evidences concerning successful construction of individual actions that become collective informative resources. This can be observed when at least two actors produce new elements, or constraints, in their communication while interacting. In other words, when one member that was not been originally trained in the language could understand it and wanted to use it, because a trained member had transferred it through their conversations. Our argument is that in this model the constraints on the communication would facilitate: a) the coordination of actions, b) the strengthening of collectives of actors who can perform some collective activity in their environments, and c) testing the effectiveness and efficiency of its collective action.

It is important to notice that both Partnerships A and B were able to develop successful strategies that currently maintain themselves alive and operating. The main distinction between both is that in Partnership A, additional internal resources have been developed. This suggests that communities that make individual actions available to others as additional resources, may become more creative through self-organization, and be able to produce a wide variety of responses as a result - in a reasonably effective and efficient manner. We propose to see these communities as a Collective Informative System, Coll-IS.

\section{Conclusion}

This paper began by describing the challenges of doing research on how individual action may become a collective informative resource. We posit that a community increases its viability when becomes a repository of such resources. We proposed to name this kind of community a 'Collective Informative System' (CoIl-IS). We consciously avoid the use of the label 
'Community Information System'. The reason is to avoid conflicts with the traditional understanding of computer-based IS. In this paper there is no claim that a traditional IS was designed or implemented (Hillier et al. 2005; Corbett and Keller 2006; Culhane and Breuer 2007). The focus was on proposing and testing a research method to discover if a community (in this case, Partnerships A and B) may act as collective informative repository for individual and collectives' actions that may better-inform their members.

Accordingly, the problem identified was of a more general nature. It involved studying Coll-IS, without being constrained to (a) the types of action to be supported, and (b) what informs particular actors, rather than any actor. This research process demands the existence of a collective operating, or its pre-organization by means of collectivizing a group of people. The latter was explored by means of developing a research project.

Throughout this paper, we intended to show that the problem is not one of a situational nature. The difficulty resides on how to maintain people together doing something, even if the original something changes. Intervened collectives cannot usually come back to their original state, so stopping and beginning again was not an option. What we propose is to recognize the richness of a community, and its effectiveness, located in the strengthening of its links, quite aside from the temporal outputs it might deliver. Hence, the focus of the research effort was on the production processes, instead of observing only outcomes or results. In view of that, this document suggests a way to develop collectives in that direction: to do something in order to do something else. In the experience presented here, the initial action was about engaging people in a process of research, to strengthen their personal links inside a collective, This was done by supporting their own reflections on what they were doing individually and how that may become informative to others' actions. In a nutshell, to reflect on 'what keeps them together doing things'. Accordingly, we decided to introduce certain notions, following ideas from Beer's Viable System Model, as a way to support and observe the processes of self-organization within the collective; to help members inside the collective (a) in recognizing different internal resources, (b) to coordinate their use, and thus (c) to behave as 'strong actors'. To define which initial something is more effective and efficient to achieve the final one is part of this research. It suggests that research may support individuals in the collective to act as reflective practitioners (Schön 1991), hoping to create in the process 'stronger actors' (de Zeeuw 2001b), meanwhile being actively engaged on self-organizing themselves as a collective.

This research design may extend to other collectives, formal and informal, where individual actions can be used by others as additional resources; in principle, this suggests a generalizable research procedure. This procedure could be used in other contexts, for other aims, such as to analyze the quality of the communicative channels in terms of accuracy, communicative closure and speed. Finally, this research approach might also involve the possibility of expanding current Information Systems research through a wider understanding; where the traditional IS elements Hardware, Software, Databases, Networks and Procedures could be map into People; their Languages, natural or constructed; their memories, individual or collective; and Communities, and their Projects and joint Activities; but this is part of another story.

\section{Appendix 1 - Proposed observational tool}

Some examples of questions from the machine are the following:

Table 6 
Table 6 Examples of questions from the linguistic machine

creation Others'meanings, externally defined amplifiers intervening results

The expected results of the collective and their operationalisation are defined by superior authorities -e.g. Home Office. These expectations are informed to their members through specific governmental procedures and regulations. These are focused on amplifying their impact by means of new technologies - e.g. internet, e-mail.
production Others' meanings,
externally defined
amplifiers
intervening
results

When producing the expected results externally defined, by operations externally designed, the members inform each other about their progress through specific governmental procedures and regulations, focused on amplifying their impact, by means of new technologies - e.g. internet, e-mail.
regulation Others' meanings,
externally defined
amplifiers
intervening
results

When evaluating the expected results, externally defined, by operations externally designed, the members are informed about the achievements through specific governmental procedures and regulations. These are focused on amplifying their impact by means of new technologies -e.g. internet, e-mail.
creation
their own meanings, externally defined
amplifiers
intervening
results

The expected results of the collective are defined by the members of the collective. However, their operationalisation must follow external guidelines -i.e. Home Office. The expectations agreed and the operations demanded are consulted and informed to their members through specific governmental procedures and regulations. These are focused on amplifying their impact by means of new technologies -e.g. internet, e-mail.

\section{creation Others'meanings, internally negotiated amplifiers intervening results}

The expected results of the collective and their operationalisation are defined by superior authorities -e.g. Home Office. These expectations are informed to their members through internally developed protocols. These are focused on amplifying their impact by means of new technologies -e.g. internet, e-mail.

creation Others'meanings, externally defined attenuators intervening results

The expected results of the collective and their operationalisation are defined by superior authorities -e.g. Home Office. These expectations are informed to their members through specific governmental procedures and regulations. These are focused on attenuating disturbances by means of new technologies -e.g. internet, e-mail.

creation Others'meanings, externally defined amplifiers responsible (for) results

The expected results of the collective are defined by superior authorities -e.g. Home Office. However, their operationalisation is let to the member's discretion. These expectations are informed to their members through externally developed protocols. These are focused on amplifying their impact by means of new technologies -e.g. internet, e-mail.

creation Others'meanings, externally defined amplifiers intervening understandings

The expected outcomes of the collective and their operationalisation are defined by superior authorities -e.g. Home Office. These expectations are informed to their members through specific governmental procedures and regulations. These are focused on amplifying their impact by means of new technologies -e.g. internet, e-mail.

Open Access This article is distributed under the terms of the Creative Commons Attribution 4.0 International License (http://creativecommons.org/licenses/by/4.0/), which permits unrestricted use, distribution, and reproduction in any medium, provided you give appropriate credit to the original author(s) and the source, provide a link to the Creative Commons license, and indicate if changes were made.

\section{References}

Ashby R (1956, 1999) An Introduction to Cybernetics. http://pespmc1.vub.ac.be/ LIBRARY.Html, accessed Nov 2002 
Axelrod R (1990) The evolution of cooperation. Penguin Books, London

Beer S (1972) Brain of the firm, 2nd edn. Wiley, The Stafford Beer Classic Library, Chichester

Beer S (1985) Diagnosing the system for organizations. Wiley, The Stafford Beer Classic Library, Chichester

Checkland P, Holwell S (1997) Information, systems and information systems. John Wiley \& Sons, Chichester

Churchman CW (1971) The Design of Inquiring Systems. Basic Books, Inc. Publishers, New York

Corbett J, Keller P (2006) Using community information systems to communicate traditional knowledge embedded in the landscape. Particip Learn Action 54:21-27

Culhane D, Breuer B (2007) The development of community information systems to support neighborhood change. In: Cnaan RA, Milofsky C (eds) Handbook of community movements and local organizations. Springer, New York

de Zeeuw G (2001a) Three phases of science: a methodological exploration. Systemica 13(1,2,3,4,5,6):433-460

de Zeeuw G (2001b) Constructivism: a 'next' area of scientific development? Found Sci Spec Issue Impact Radic Constructivism Sci 6(1-3):77-98

de Zeeuw G (2010) Research to support social interventions. J Soc Interv: Theory Prac 19(2):4-24

Dewey J (1948) Sense and science: their respective frames of reference. J Philos 45(8):197-208

Espejo R (2000) Self-construction of desirable social systems. Kybernetes 29(7/8):949-964

Espejo R, Schuhmann W, Schwaninger M, Bilello U (1996) Organizational transformation and learning. Wiley, Chichester

Fischhoff B (1992) Giving advice: decision theory perspectives on sexual assault. Am Psychol 47(4):577-588

Guttman L (1959) A structural theory of intergroups beliefs and action. Am Sociol Rev 24:318-328

Hillier AE, Wernecke ML, McKelvey H (2005) Removing barriers to the use of community information systems. J Community Pract 13(1):121-139

Home Office (1998) Crime and Disorder Act 1998, Chapter 37. [online] http://www.legislation.gov. uk/ukpga/1998/37/contents, [accessed 26 Nov 2016]

Hoverstadt P, Bowling D (2002) Modelling organizations using the viable system model. Royal Academy of Engineering Systems Engineering Workshop, May 23 ${ }^{\text {rd }}$ 2002. [online] http://www.scio.org. uk/sites/default/files/VSM_ph_db.pdf, [accessed 16 Nov 2016]

Kast FE, Rosenzweig JE (1985) Organization \& Management: a systems and contingency approach, 4th revised ed. McGraw-Hill, Inc, New York

Lave J, Wenger E (1991) Situated learning: legitimate peripheral participation. University of Cambridge Press, Cambridge

Luhmann, N (1984) Soziale Systeme: grundisse einer Allgemeinen Theorie. Frankfurt am Main: Suhrkamp Verlag. Translated to Spanish (1991) Sistemas Sociales: Lineamientos para una teoría general. México: Editorial Patria / Universidad Iberoamericana

Ostrom E (2009) Beyond markets and states: polycentric governance of complex economic systems. Nobel Prize Lecture, December 8, 2009. [online] https://www.nobelprize.org/nobel_prizes/economicsciences/laureates/2009/ostrom_lecture.pdf. [accessed 6 April 2017]

Rapoport A (1988) Experiments with n-person social traps II: tragedy of the commons. J Confl Resolut 32(3): $473-488$

Schön D (1991) The reflective practitioner: how professionals think in action. Avebury, Aldershot

Searle JR (1996) The construction of social reality. Penguin Books, London

Searle JR (2005) What is an institution? J Inst Econ 1(1):1-22

Sen A (2005) Why exactly is commitment important for rationality? Econ Philos 21:5-14

Shye S (1978) On the search for laws in the behavioral sciences. In: Shye S (ed) Theory construction and data analysis in the behavioral sciences. Jossey-Bass Publishers, San Francisco, pp 2-24

Simon H (1957) A behavioral model of rational choice. In: Models of man, social and rational: mathematical essays on rational human behavior in a social setting. Wiley, New York

Skyrms B (2007) Evolution and the social contract. The Tanner Lectures on Human Values. The University of Michigan. [online] http://tannerlectures.utah.edu/_documents/a-to-z/s/Skyrms_07.pdf

Spencer-Brown G (1972) Laws of form. Cognizer Company, Portland

Taylor F (1911) The Principles of Scientific Management. [online] available at https://archive. org/details/principlesofscie00taylrich [accessed 1st of Oct 2014]

Ulrich W (2005) A brief introduction to critical systems heuristics (CSH). Web site of the ECOSENSUS project, Open University, Milton Keynes, UK. [online] available at http://www.ecosensus.info/about/index.html [accessed 13th of Nov 2017]

von Foerster H (1999) Las Semillas de la Cibernética. Gedisa Editorial, Barcelona 